\title{
Identification of Legionella Pneumophila in Intubated Patients With TaqMan Real Time PCR
}

\author{
Nader Divan Khosroshahi ${ }^{1}$; Taghi Naserpour Farivar ${ }^{1, *} ;$ Pouran Johari $^{1}$ \\ ${ }^{1}$ Cellular and Molecular Research Centre, Qazvin University of Medical Sciences, Qazvin, IR Iran \\ ${ }^{*}$ Corresponding author: Taghi Naserpour Farivar, Cellular and Molecular Research Centre, Qazvin University of Medical Sciences, Qazvin, IR Iran. Tel: +98-9128801401, Fax: +98- \\ 2833324971, E-mail: taghin@yahoo.com
}

Received: September 28, 2013; Revised: June 7, 2014; Accepted: June 14, 2014

\begin{abstract}
Background: Legionellaceae contains Legionella genus with over 52 species and 64 serogroups. It is one of the most important causes of respiratory disease in human. More than 30\% of hospital-acquired pneumonia is caused by Legionella. Ventilator-associated pneumonia (VAP) is an infection acquired in hospital wards, particularly in intensive care unit (ICU). This disease approximately affects $9 \%$ to $20 \%$ of intubated patients. Mortality in these patients varies between $8 \%$ and $76 \%$. Legionella is one of the important factors for infection in intubated patients.

Objectives: The present study was aimed to investigate the use of molecular methods in diagnosis of infection caused by Legionella pneumophila.

Materials and Methods: In this study, 109 samples of lung secretions collected from intubated patients admitted to ICU wards of four university hospitals in a three-month period were examined. Cultivation and Real time Polymerase Chain Reaction (PCR) methods were used to assess L. pneumophila colonization in these samples.

Results: Eleven samples had positive results using real time PCR analysis of 16s rRNA gene fragments specific for L. pneumophila, but according to culture method on specific buffered charcoal-yeast extract medium (BCYE), no positive cases were detected. Of the total positive cases, six were males, one female and four infants. The seven adults aged 40-65 years.

Conclusions: Using molecular methods in diagnosis of infection caused by L.pneumophila has a great value because of its high specificity and rapid diagnosis potency.
\end{abstract}

Keywords:Legionella pneumophila; Real Time Polymerase Chain Reaction; Cross Infection; Patients

\section{Background}

Legionellaceae family contains only one genus Legionella and over 52 species and 64 serogroups, which is one of the most important causes of respiratory disease in humans. The bacteria are abundant in man-made aquatic environments and water resources (1). The most important species of this genus is Legionellaceae pneumophila, which has 15 serogroups. L. pneumophila is operating more than $90 \%$ of the legionary, which is an acute respiratory disease. Serogroups 1 and 6 are the causes of two thirds of cases of Legionella infection (2-4). Mortality rate in elderly and immunocompromised patients with L. pneumonia may be more than $30 \%(3,4)$.

Various reports suggest that $1-5 \%$ of community-acquired pneumonia as well as over $30 \%$ of hospital-acquired pneumonia infections are caused by Legionella (5). Children younger than two years or elderly adults and patients with immunodeficiency are at highest risk of infection. The infection is not contagious and only a breath of aerosols contaminated with this bacterium may cause infection and clean air is an important parameter in the prevention of respiratory infection with this bacterium. Considering clinical signs and symp- toms of disease, L. pneumonia cannot be differentiated from other pneumonia (6). Timely diagnosis and treatment of infection disease is effective in reducing the mortality rate. Epidemiological findings indicate that this bacterium is transmitted through aerosols released of infected water sources and involves the respiratory system. Hospital environment as a growth area and people at risk of aerosol transmission are potential predisposing factors for growth and spread of these bacteria. Legionella are widely dispersed in natural and manmade water sources (7). Legionnaire's outbreak depends on the contamination of water sources and sensitivity of individuals (2).

Cultivation methods, serological tests, detection of specific antigen in urine and molecular detection methods can be used to identification and detection of Legionnaires' disease. Although culture method like other bacteria is the gold standard for detection of these bacteria, the use of this method is limited for the diagnosis of infection in patients, because the bacterium needs at least seven days to appear colonies $(2,8)$. Serological tests for diagnosis of Legionella infections are a valuable

Copyright (C) 2015, Ahvaz Jundishapur University of Medical Sciences. This is an open-access article distributed under the terms of the Creative Commons Attribution-NonCommercial 4.0 International License (http://creativecommons.org/licenses/by-nc/4.0/) which permits copy and redistribute the material just in noncommercial usages, provided the original work is properly cited. 
epidemiological tool, but not useful for rapid detection of acute cases of Legionnaires' disease (9). Direct Fluorescent Antibody assays (DFA) on respiratory samples of patients or urinary antigens is a rapid diagnostic method, but using this method is limited due to cross reactions with other bacteria (6). The use of molecular methods in diagnosis of Legionnaires' disease has very high diagnostic sensitivity, specificity and speed (10). The genes encoding $5 \mathrm{~s}$ and 16s rRNA and macrophage infectivity potentiator ( $\mathrm{mip}$ ) gene are often very important for the detection of L. pneumonia, Therefore, the target sequences of these genes are useful to determine the genus Legionella and the species of L. pneumophila (10, 11). Diagnosis of Legionnaires' disease and choosing the correct treatment can be very effective in the treatment and prevention of mortality in patients. Due to high specificity and characteristics of molecular detection methods, in this study, in addition to culture methods, the new technique was used for detection of L. pneumophila -positive cases.

\section{Objectives}

The aim of this study was to investigate the use of molecular methods in diagnosis of infection caused by $L$. pneumophila.

\section{Materials and Methods}

In this study, 109 samples of lung secretions were collected from intubated patients admitted to ICU (Intensive Care Unit) and NICU (Neonatal Intensive Care Unit) wards of four hospitals affiliated to the University of Qazvin (Bu Ali, Quds, Kosar and Shahid Rajaie hospitals) in a three-month period. The secretions were divided into two parts. One part used for cultivation and the other for molecular testing. The samples of molecular testing kept in freezer at $-20^{\circ} \mathrm{C}$ until testing.

\subsection{Culture on Buffered Charcoal-yeast Extract (BCYE) Agar}

The samples were placed in washing buffer acid (HCL / $\mathrm{KCL}, \mathrm{PH} 2.2$ ) at $56^{\circ} \mathrm{C}$ to remove the annoying factor (10). Then, samples were inoculated on specific BCYE medium (OXOID; UK). The plates incubated for a week at $37^{\circ} \mathrm{C}$ with humidity maintained and with $5 \% \mathrm{CO}_{2}$. Colonies appeared after the third day. We prepared a microscopic slide using Gram-staining method. We cultured suspect colony on the routine laboratory media. Nevertheless, Legionella did not grow on it. Then, we performed biochemical tests such as catalase, oxidase, B lactamase, hyporate and other standard bacteria biochemistry tests for the identification of Legionella (1). L. pneumophila ATCC33152 was used as a control standard.

\subsection{Internal DNA Extraction Control}

Extraction was performed according to kit instruc- tions (Primerdesign, UK) according to the manufacturer's instructions. All samples after the addition of lyses' buffer were heated at $95^{\circ} \mathrm{C}$ for 30 minutes and the presence of extracted DNA in samples was measured by amplification of Actin Beta (ACTB) gene using supplied primer and probe (Primerdesign, UK). For this purpose, we used the following values; $10 \mu \mathrm{L}$ of RT-PCR (Reverse transcription polymerase chain reaction) Master mix, 1 $\mu \mathrm{L}$ of the ACTB primer/probe solution, $4 \mu \mathrm{L}$ of distilled water free of RNAse/DNAse and $5 \mu \mathrm{L}$ of the sample. Real time PCR amplification reaction was performed in Applied Bio system 7500 with the following schedule; $95^{\circ} \mathrm{C}$ for 10 minutes; 40 cycles at $95^{\circ} \mathrm{C}$ for 15 seconds and $60^{\circ} \mathrm{C}$ for one minute. A poor ACTB signal may indicate that the sample did not contain sufficient biological material.

\subsection{Real Time PCR}

TaqMan Real time PCR experiments were performed according to the manufacturer's instructions (Advanced kit L. Pneumophila Primer design, UK), which would identify the 16 s ribosomal gene in L. pneumonia species. The following amounts were added to each reaction; 10 $\mu \mathrm{L}$ of RT-PCR Master mix, $1 \mu \mathrm{L}$ of the primer/probe, $4 \mu \mathrm{L}$ of distilled water free of RNAse/DNAse and $5 \mu \mathrm{L}$ of the samples from patients. After 10 minutes of denaturation at $95^{\circ} \mathrm{C}$, Polymerase chain reaction was run for 40 cycles at $95^{\circ} \mathrm{C}$ for 15 seconds and $60^{\circ} \mathrm{C}$ for 60 seconds in Applied Bio system 7500.

\section{Results}

In total, 109 samples of lung secretions were collected from patients admitted to Qazvin University of Medical Sciences's Hospitals (55 samples from Shahid Rajai Hospital, 19 samples from Bu Ali Hospital, 19 samples from Kosar hospital and 16 samples from Quds hospital). From these collected samples, six samples had negative results for ACTB assay and were excluded from the study. Amplification curve of the standard sample with different concentrations were provided (Figure 1). Using different concentrations of control, with decreasing diluents, sample A was shown in higher cycle (CT of A, B and C were respectively 15, 22, 25). From the remaining 103 samples, 11 samples (11.99\%) had positive results for L. pneumonia by real time PCR method (Figure 2), while no positive samples were detected using the culture method on BCYE medium. All cases were the positive L. pneumophila isolated, six cases (10.9\%) from Shahid Rajai Hospital, four cases (25\%) from Quds hospital and one case (5.3\%) from Bu-Ali Hospital and no positive cases were found among samples taken from Kosar Hospital. Of the total positive cases, six were males and one female; while we had four positive results among our infants samples. Seven positive results belonged to patients aged 40-65 years. According to our results, 7 of 11 positive samples had been more than oneweek in hospital and had positive results in the second sampling (Table 1). 


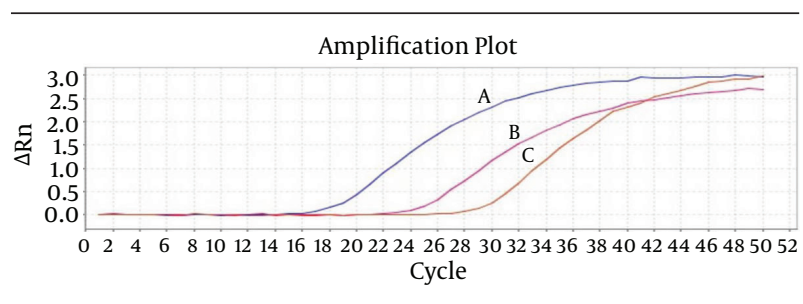

Figure 1. Amplification Curve of Standard Samples With Concentrations of A, $5 \times 104$, B, $5 \times 102, C, 5 \times 101$ copy per micro litter

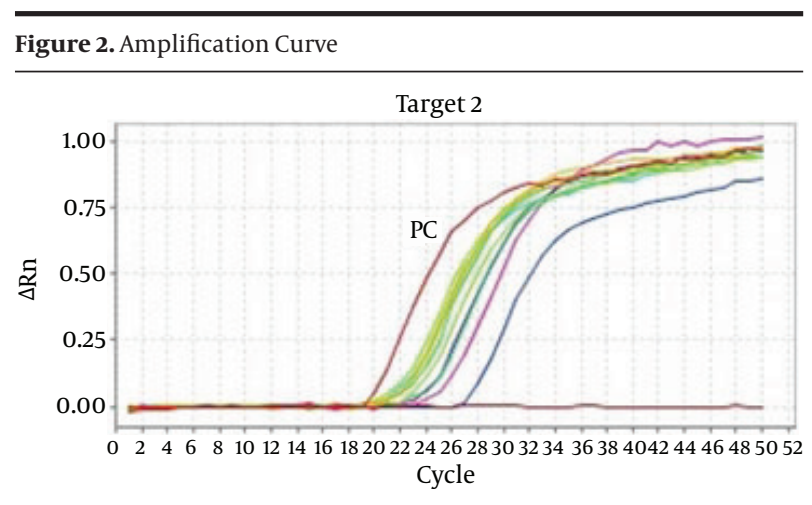

PC; positive control and positive patients.

Table 1. Total Sampling and Positive Results by Real Time PCR

\begin{tabular}{lccccc}
\hline Hospitals & Buali & Rajai & Qods & Kosar & Total \\
\hline Samples & 19 & 55 & 16 & 19 & 109 \\
Culture on BCYE & 0 & 0 & 0 & 0 & 0 \\
Real time PCR & 1 & 6 & 4 & 0 & 11 \\
Percent & 5.3 & 10.9 & 25 & 0 & 11.99 \\
\hline
\end{tabular}

\section{Discussion}

Hospital acquired pneumonia (HAD) is pneumonia that develops 48 hours or longer after admission to hospital. HAP is the second most common nosocomial infection. Ventilator-associated pneumonia (VAP) is an infection acquired in hospital wards, particularly in ICU. Ventilatorassociated pneumonia (VAP) is pneumonia that develops 48 hours or longer after mechanical ventilation is given by means of an endotracheal tube or tracheostomy. Ventilator-associated pneumonia (VAP) results from the invasion of lower respiratory tract and lung parenchyma by microorganisms. This disease affects approximately $9 \%$ to $20 \%$ of intubated patients. Mortality rate in these patients varies between $8 \%$ and $76 \%(12,13)$. VAP is caused by different factors and in most cases, causing agents are bacteria not readily cultivated on conventional media, and if not quickly diagnosed and appropriate treatment, the mortality rate increases in patients.

Studies showed that Legionella can cause one third of hospital pneumonia and the mortality rate of infection is approximately $5 \%$ to $30 \%$ and is more in elderly and im- munocompromised patients (14). Most Legionnaires' cases, approximately $90 \%$, is caused by L. pneumophila (15). Therefore, extensive research is performed on identifying and controlling the genus Legionella. This infection is caused due to contact with a water contaminant source. Legionnaires' disease often occurs in sporadic outbreaks and sporadic form of disease is between $65-82 \%$ (16). Legionnaires disease has increased in recent decades. One reason may be development of water systems such as air conditions, cooling towers, ponds, water storage resources in hospitals, artificial ponds and steam generation systems, which have a role in maintenance and transmission of Legionella. Early diagnosis of infection plays an important role in the treatment and prevention of mortality in patients (4).

Although the culture method is the gold standard technique, studies showed that the sensitivity of culture methods in samples from patients has been less than 50$60 \%$ (2). Many studies showed that the use of molecular methods in diagnosis of infection caused by L. pneumophila has a great value because of its high specificity and rapid diagnosis of disease (17). Previous studies suggested that the culture method is not efficient for detecting bacteria in environmental samples and in clinical samples. In a study performed on water samples collected from a hotel, $42 \%$ of the samples had positive results using culture, while it was $74 \%$ by real time PCR techniques (18). In another study conducted on water resources, of 120 samples, 31 samples (26\%) had positive results by culture method, while 57 samples (47.5\%) had positive findings in real time PCR method (19). In a similar study performed in Japan, of 208 respiratory samples of patients with community acquired pneumonia (CAP) using multiplex Real time PCR method, eight samples (6.16\%) had positive results for $L$. pneumophila, but using the culture method, only one patient was detected (20). In another study performed on 60 samples taken from respiratory secretions in intubated patients admitted to ICU, three cases were detected by PCR (9).

Results of this research also confirmed that the culture method for detection of Legionella infection has a low efficiency and this infection can be better investigated using molecular diagnostic methods. The reason for this difference in culture results and molecular diagnostic methods could be due to the fact that molecular diagnostic methods can recognize all cells whether alive or dead, but in the culture method, only viable cells can be detected. Furthermore, bacteria concentration in the culture method is effective in its isolation; however, molecular methods can detect the presence of even one bacterium. Considering that late diagnosis of infection can increase the mortality rate, rapid detection of bacteria can be very helpful in the process of more efficient treatment (2). Results obtained in this study showed that most positive cases belonged to the Quds hospital with 25\% (4 of 16 samples). Considering that two cases of patients were diagnosed in one day and two other cases isolated 
in a small period, it can be concluded that infection is likely to originate from an infected water source. We recommend ICU and NICU departments in medical centers, especially hospitals, to consider infection control and prevention more seriously.

\section{Acknowledgements}

We would like to thank all laboratory staff in Qazvin University of Medical Sciences and Cellular and Molecular Research center.

\section{Funding/Support}

This study was supported by Qazvin University of Medical Sciences.

\section{References}

1. Baron EJF, Finegold SM. Diagnostic microbiology. 8th edNew York: USA: Mosby company; 1990.

2. Bonetta S, Bonetta S, Ferretti E, Balocco F, Carraro E. Evaluation of Legionella pneumophila contamination in Italian hotel water systems by quantitative real-time PCR and culture methods. $J$ Appl Microbiol. 2010;108(5):1576-83.

3. Mandell GL, Bennett JE, Dolin R. Principles and practice of infectious disease.Philadelphia: USA: Churchill Livingstone; 2000.

4. Yu VL, Plouffe JF, Pastoris MC, Stout JE, Schousboe M, Widmer A, et al. Distribution of Legionella species and serogroups isolated by culture in patients with sporadic community-acquired legionellosis: an international collaborative survey. J Infect Dis. 2002;186(1):127-8.

5. Berger P, Papazian L, Drancourt M, La Scola B, Auffray JP, Raoult D. Ameba-associated microorganisms and diagnosis of nosocomial pneumonia. Emerg Infect Dis. 2006;12(2):248-55.

6. Shadrach WS, Rydzewski K, Laube U, Holland G, Ozel M, Kiderlen $\mathrm{AF}$, et al. Balamuthia mandrillaris, free-living ameba and opportunistic agent of encephalitis, is a potential host for Legionella pneumophila bacteria. Appl Environ Microbiol. 20 05;71(5):2244-9.

7. Reischl U, Linde HJ, Lehn N, Landt O, Barratt K, Wellinghausen N. Direct detection and differentiation of Legionella spp. and Legionella pneumophila in clinical specimens by dualcolor real-time PCR and melting curve analysis. J Clin Microbiol. 2002;40(10):3814-7.

8. Den Boer JW, Yzerman EP. Diagnosis of Legionella infection in Legionnaires' disease. Eur J Clin Microbiol Infect Dis. 2004;23(12):8718.

9. Mokhless NAS, El-Mofty MF, Hanafi NF, Muhammad A. Atypical Bacteria in Ventilator Associated Pneumonia; an Egyptian University Hospital Experience. J American Sci. 2010;6(12).

10. Wilson DA, Yen-Lieberman B, Reischl U, Gordon SM, Procop GW. Detection of Legionella pneumophila by real-time PCR for the mip gene. J Clin Microbiol. 2003;41(7):3327-30.

11. van der Eerden MM, Vlaspolder F, de Graaff CS, Groot T, Jansen HM Boersma WG. Value of intensive diagnostic microbiological investigation in low- and high-risk patients with community-acquired pneumonia. EurJ Clin Microbiol Infect Dis. 2005;24(4):241-9.

12. Tan B, Zhang F, Zhang X, Huang YL, Gao YS, Liu X, et al. Risk factors for ventilator-associated pneumonia in the neonatal intensive care unit: a meta-analysis of observational studies. Eur J Pediatr. 2014;173(4):427-34.

13. De Souza PR, De Andrade D, Cabral DB, Watanabe E. Endotracheal tube biofilm and ventilator-associated pneumonia with mechanical ventilation. Microsc Res Tech. 2014;77(4):305-12.

14. Eryuksel E, Karakurt S, Balci M, Celikel T. Non-invasive positive pressure ventilation for a severe legionella pneumonia case. $\mathrm{Tu}$ berk Toraks. 2009;57(3):348-51.

15. Newton HJ, Ang DK, van Driel IR, Hartland EL. Molecular pathogenesis of infections caused by Legionella pneumophila. Clin Microbiol Rev. 2010;23(2):274-98.

16. Joseph CA, European Working Group for Legionella I. Legionnaires' disease in Europe 2000-2002. Epidemiol Infect. 2004;132(3):417-24.

17. Wellinghausen N, Frost C, Marre R. Detection of legionellae in hospital water samples by quantitative real-time LightCycler PCR. Appl Environ Microbiol. 2001;67(9):3985-93.

18. Benson RF, Fields BS. Classification of the genus Legionella. Semin Respir Infect. 1998;13(2):90-9.

19. Cianciotto NP. Pathogenicity of Legionella pneumophila. Int Med Microbiol. 2001;291(5):331-43.

20. Miyashita N, Saito A, Kohno S, Yamaguchi K, Watanabe A, Oda $\mathrm{H}$, et al. Multiplex PCR for the simultaneous detection of Chlamydia pneumoniae, Mycoplasma pneumoniae and Legionella pneumophila in community-acquired pneumonia. Respir Med. 2004;98(6):542-50. 